\title{
Near-ideal van der Waals rectifiers based on all-two-dimensional Schottky junctions
}

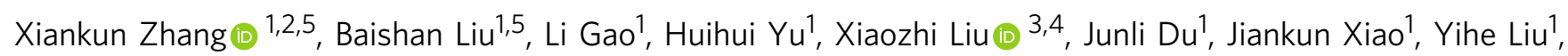

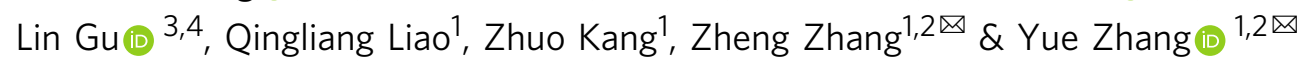

The applications of any two-dimensional (2D) semiconductor devices cannot bypass the control of metal-semiconductor interfaces, which can be severely affected by complex Fermi pinning effects and defect states. Here, we report a near-ideal rectifier in the all-2D Schottky junctions composed of the $2 \mathrm{D}$ metal $1 \mathrm{~T}^{\prime}-\mathrm{MoTe}_{2}$ and the semiconducting monolayer $\mathrm{MoS}_{2}$. We show that the van der Waals integration of the two 2D materials can efficiently address the severe Fermi pinning effect generated by conventional metals, leading to increased Schottky barrier height. Furthermore, by healing original atom-vacancies and reducing the intrinsic defect doping in $\mathrm{MoS}_{2}$, the Schottky barrier width can be effectively enlarged by $59 \%$. The $1 \mathrm{~T}^{\prime}-\mathrm{MoTe}_{2}$ /healed-MoS 2 rectifier exhibits a near-unity ideality factor of $\sim 1.6$, a rectifying ratio of $>5 \times 10^{5}$, and high external quantum efficiency exceeding $20 \%$. Finally, we generalize the barrier optimization strategy to other Schottky junctions, defining an alternative solution to enhance the performance of 2D-material-based electronic devices.

\footnotetext{
${ }^{1}$ Beijing Advanced Innovation Center for Materials Genome Engineering, Beijing Key Laboratory for Advanced Energy Materials and Technologies, University of Science and Technology Beijing, Beijing, People's Republic of China. ${ }^{2}$ State Key Laboratory for Advanced Metals and Materials, School of Materials Science and Engineering, University of Science and Technology Beijing, Beijing, People's Republic of China. ${ }^{3}$ Collaborative Innovation Center of Quantum Matter, Beijing, China. ${ }^{4}$ Beijing National Laboratory for Condensed Matter Physics, Institute of Physics, Chinese Academy of Sciences, Beijing, China. ${ }^{5}$ These authors

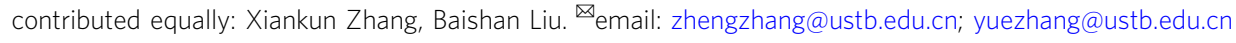


M etal-semiconductor interfaces are crucial to the operations and applications of every semiconductor devices ${ }^{1}$, and are at the basis of Schottky diodes. With excellent on-off switching characteristics, Schottky diodes often replace $\mathrm{p}-\mathrm{n}$ diodes in many essential components of modern electronics and optoelectronics ${ }^{2-5}$. Recently, without the constraint limits of lattice matching and processing compatibility requirements of conventional bonded heterojunctions, two-dimensional (2D) van der Waals (vdWs) heterojunctions have attracted widespread interests $^{6-10}$. To date, the traditional merits of Schottky diodes are not reproduced in 2D material-based Schottky diodes but are replaced by many deficiencies, such as excessive reverse leakage current (i.e., large power consumption), low current rectifying ratio, and poor ideality factor ${ }^{4,11-15}$. This is attributed to the composition effect of the low Schottky barrier height and the tiny Schottky barrier width in 2D Schottky diodes in Fig. 1a, which are originated respectively from the strong Fermi pinning effect of the metal-semiconductor junctions and the ultrathin body thickness ${ }^{2,5,16-20}$.

Both achieving the designed Schottky barrier height and precisely controlling the barrier width are crucial for realizing the full potential of Schottky diodes. For Schottky barrier height, transferring metals or employing 2D metals can build weak Fermi pinned Schottky junctions to roughly satisfy the personalized barrier height ${ }^{2,21-23}$. The $2 \mathrm{D}$ metals (e.g., $1 \mathrm{~T}^{\prime}-\mathrm{MoTe}_{2}, 1 \mathrm{~T}^{\prime}-\mathrm{WTe}_{2}$, $1 \mathrm{~T}^{\prime}-\mathrm{PtSe}_{2}$, and $2 \mathrm{H}-\mathrm{NbSe}_{2}$ ), by contrast, have more potential in creating high-grade Schottky junctions because their Schottky barrier heights strictly follow the trend of the Schottky-Mott model. Since 2D metals can form the weaker chemical bonding interface with $2 \mathrm{D}$ vdWs semiconductors and efficiently suppress the metal-induced gap states in conventional Schottky junctions ${ }^{22,24,25}$. The metal-induced gap states are one of the important sources of the Fermi pinning effect ${ }^{2}$.

On the other hand, the tunneling currents widely exist in the 2D semiconductor-based Schottky junctions ${ }^{18}$, which is highly related to its tiny Schottky barrier widths and will form the large leakage currents to cause the high power consumptions of diodes. To date, the adjustments of the Schottky barrier widths often rely on surface absorption chemical doping, which is environment unstable and inefficient in controlling the electronic structures $^{26,27}$. Lattice defect healing is an optimal strategy to efficiently and stably decrease the intrinsic defect doping of ultrathin $2 \mathrm{D}$ materials ${ }^{28,29}$.

Here, we report a near-ideal Schottky diode in $1 \mathrm{~T}^{\prime}-\mathrm{MoTe}_{2} /$ $\mathrm{MoS}_{2}$ Schottky junction composed of the metallic $1 \mathrm{~T}^{\prime}-\mathrm{MoTe}_{2}$ and the semiconducting monolayer $\mathrm{MoS}_{2}$ in Fig. 1b. We show constructing all 2D Schottky junctions can greatly reduce the Fermi pinning effect in the metal-semiconductor interfaces. Furthermore, we firstly demonstrate that by healing original defects, shrinking the intrinsic defect doping in monolayer $\mathrm{MoS}_{2}$ can effectively enlarge the Schottky barrier width to significantly suppress the reverse tunneling leakage current in Fig. 1c. After the defect healing in monolayer $\mathrm{MoS}_{2}$, the $1 \mathrm{~T}^{\prime}-\mathrm{MoTe}_{2}$ /healed-MoS Schottky diode shows a near-unity ideality factor of $\sim 1.6$, a high rectifying ratio of $>5 \times 10^{5}$ in monolayer semiconductor-based Schottky diodes to date, and high external quantum efficiency of $>20 \%$. The Schottky barrier optimization strategy is generally applicable to other Schottky junctions, including $\mathrm{Pd} / \mathrm{MoS}_{2}$, $1 \mathrm{~T}^{\prime}-\mathrm{MoTe}_{2} / \mathrm{WS}_{2}, 1 \mathrm{~T}-\mathrm{PtSe} \mathrm{S}_{2} / \mathrm{MoS}_{2}$, and $1 \mathrm{~T}-\mathrm{PtSe} \mathrm{S}_{2} / \mathrm{WS}_{2}$. This work provides an alternative solution for improving the performance of 2D material-based Schottky junctions.

\section{Results}

Basic characterizations of the Schottky junctions. The idea and strategy of the Schottky junction optimization are shown in Fig. 1a-c. The detailed differences between the general and optimized Schottky junctions by the conventional and 2D metals are summarized in Fig. 1a, b. A monolayer $\mathrm{MoS}_{2}$ transistor with symmetric $1 \mathrm{~T}^{\prime}-\mathrm{MoTe}_{2}$ electrodes in Fig. 2a was built to characterize the Schottky barrier height between monolayer $\mathrm{MoS}_{2}$ and $1 \mathrm{~T}^{\prime}-\mathrm{MoTe}_{2}$. The monolayer $\mathrm{MoS}_{2}$ and few-layer $1 \mathrm{~T}^{\prime}-\mathrm{MoTe}_{2}$ are chemical vapor a General Schottky junction
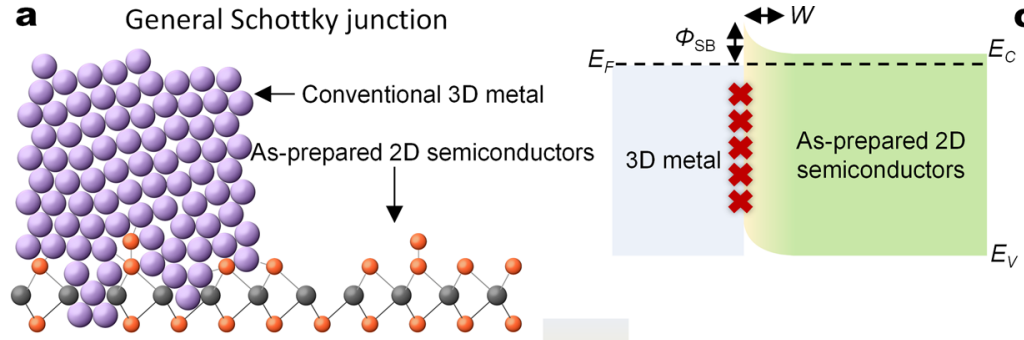

Increasing

$\Phi_{\mathrm{SB}}$ and $W$

b Optimized Schottky junction
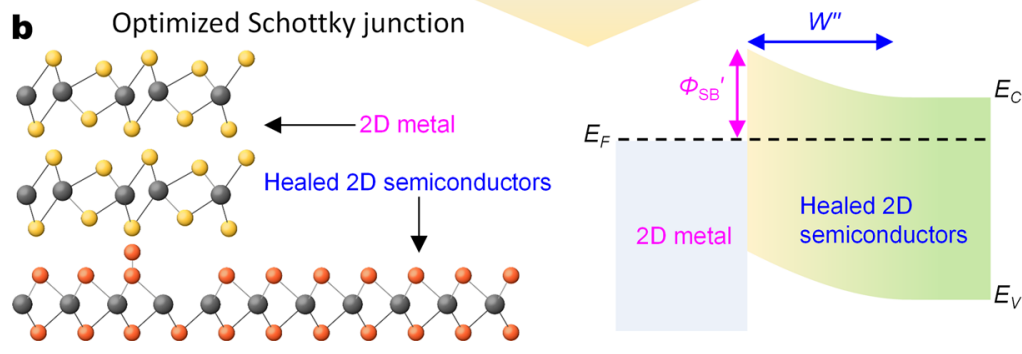

C General
Schottky junctio

Optimized Schottky junction

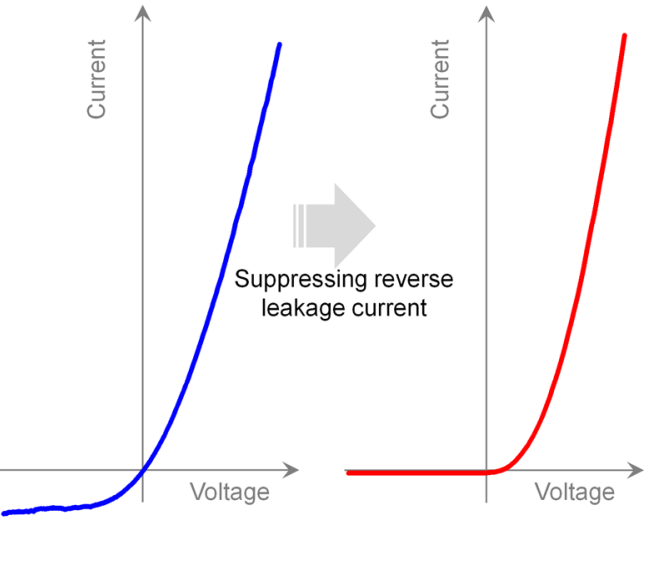

Fig. 1 Design and novelties of all 2D Schottky junctions for ideal rectifiers. a, b Schematic diagrams of atomic structure (left) and band structure (right) of general (a) and optimized (b) Schottky junctions. The purple sphere is the element of the conventional metal electrode, and the yellow, orange, and gray spheres are the composed atoms of 2D metals and semiconductors, respectively. The red cross suggests the Fermi pinning effect. The yellow gradient areas refer to the depletion region of Schottky junction. Both the lattice defects involved by the evaporation of conventional two-dimensional (3D) metals, the decaying metallic wavefunction of conventional metals, and the interface states will cause the severe Fermi pinning effect which results in the small Schottky barrier height $\Phi_{\mathrm{SB}}$. On the other hand, the intrinsic defect doping in as-prepared 2D semiconductors will render the high carrier concentration, which brings about the tiny Schottky barrier width $W$. The small height $\Phi_{S B}$ and tiny width $W$ of Schottky barrier together will lead to the large reverse leakage current of the Schottky diode. c Current-voltage curves of general (left) and optimized (right) Schottky junctions. 
a
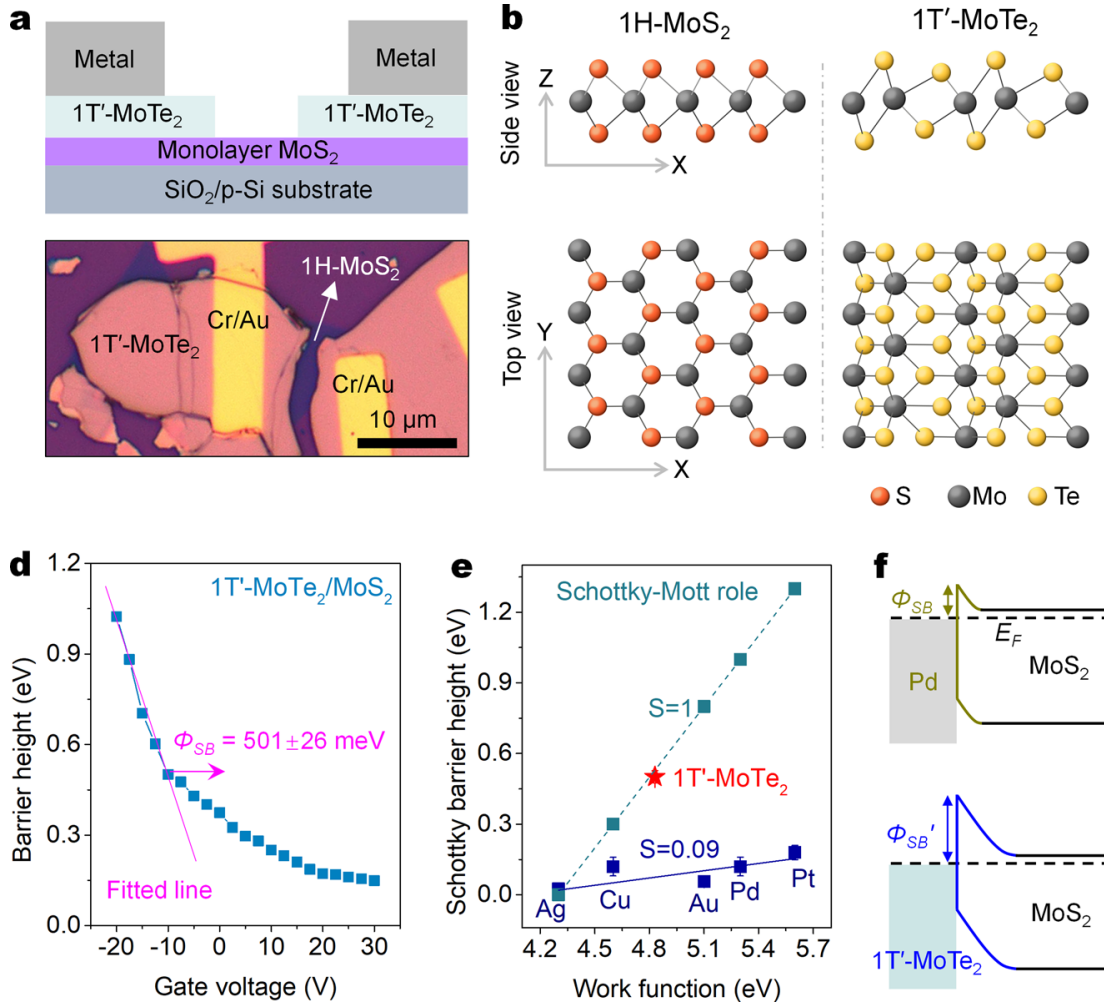

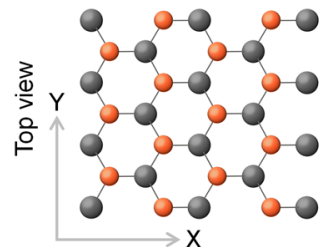

b $\quad 1 \mathrm{H}-\mathrm{MoS}_{2}$
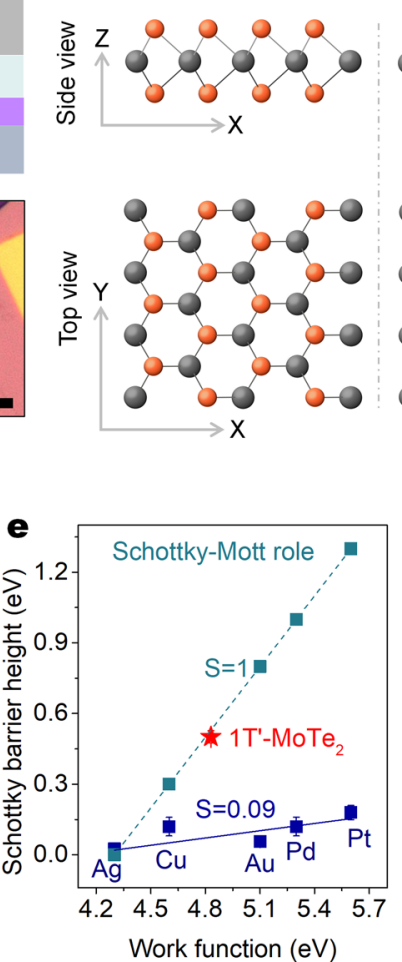

OS OMo Te

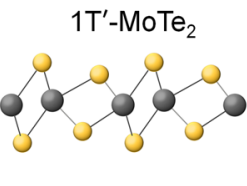

f
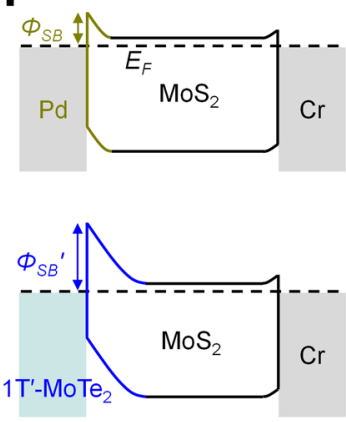

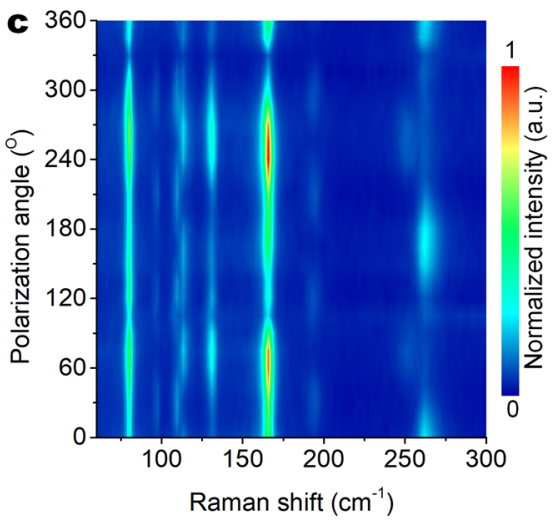

g

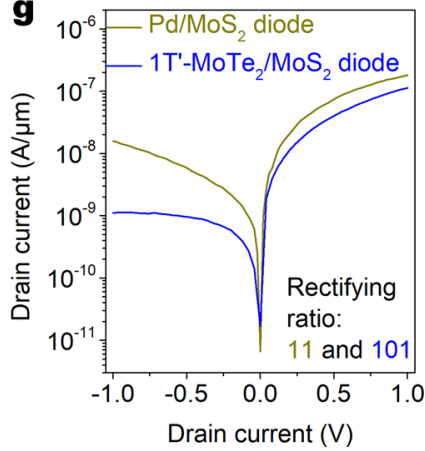

Fig. 2 The $1 \mathbf{1 T}^{\prime}-\mathrm{MoTe}_{2} / \mathrm{MoS}_{\mathbf{2}}$ Schottky junction with large Schottky barrier height and weak Fermi pinning effect. a Schematic diagram (top) and optical micrograph (bottom) of a monolayer $\mathrm{MoS}_{2} \mathrm{FET}$ with symmetric $1 \mathrm{~T}^{\prime}-\mathrm{MoTe}_{2}$ electrodes. b Crystal structures of the $1 \mathrm{H}-\mathrm{MoS}_{2}$ (left) and $1 \mathrm{~T}^{\prime}-\mathrm{MoTe}_{2}$ (right) monolayers. c Angle-resolved Raman spectra of the mechanically exfoliated $1 \mathrm{~T}^{\prime}-\mathrm{MoTe}_{2}$ flake. d Barrier heights of the $1 \mathrm{~T}^{\prime}-\mathrm{MoTe}_{2} / \mathrm{MoS}_{2} \mathrm{Schottky} \mathrm{junction}$ as a function of gate voltage obtained from Supplementary Fig. 4. The Schottky barrier height is extracted under a flat band gate voltage condition, which is responsible for the start of deviations from the linear behavior. e Comparisons of the extracted Schottky barrier height (SBH) between monolayer MoS 2 and the electrodes of evaporated 3D metals (extracted from ref. ${ }^{2}$ ) and the $2 \mathrm{D}$ metal $1 \mathrm{~T}^{\prime}-\mathrm{MoTe}_{2}$. The gunmetal dash line and points are the calculated SBH from the ideal Schottky-Mott model. The blue solid line is fitted line of experimental data. $\mathbf{f}, \mathbf{g}$ Band diagrams (f) and output curves ( $\mathbf{g}$ ) of the monolayer $\mathrm{MoS}_{2}$ diodes with the $\mathrm{Pd}$ and $1 \mathrm{~T}^{\prime}-\mathrm{MoTe}_{2}$ electrodes. All gate voltages are $\mathrm{OV}$.

deposition (CVD) grown and mechanically exfoliated ('Methods'), respectively. The $1 \mathrm{H}-\mathrm{MoS}_{2}$ has a trigonal prismatic structure and lacks inversion symmetry; while the $1 \mathrm{~T}^{\prime}-\mathrm{MoTe}_{2}$ has a distorted octahedral structure and inversion symmetry in Fig. 2b. The intensities of the several modes (e.g., $194 \mathrm{~cm}^{-1}$ ) exhibit a four-lobed shape with four maximum-intensity angles at $\sim 10^{\circ}, \sim 100^{\circ}, \sim 190^{\circ}$, and $\sim 280^{\circ}$ in Fig. $2 \mathrm{c}$ and Supplementary Fig. 1. This data conform to the polarization dependence of the Raman signals of $1 \mathrm{~T}^{\prime}-\mathrm{MoTe}_{2}$ films ${ }^{30}$. Besides, none of the relevant Raman spectra about $2 \mathrm{H}-$ $\mathrm{MoTe}_{2} \quad\left(A_{1 g} / E_{2 g}: \quad 170 / 231 \mathrm{~cm}^{-1}\right)$ are observed (Supplementary Fig. $2 \mathrm{~b}$ ), indicating that the material fabrication process did not induce a $1 \mathrm{~T}^{\prime}$-to- $2 \mathrm{H}$ phase transition ${ }^{12,31}$.

Characterization of Schottky barrier height. Kelvin probe force microscopy (KPFM) was used to characterize the work function of the metallic $1 \mathrm{~T}^{\prime}-\mathrm{MoTe}_{2}$ nanosheet as $\sim 4.83 \mathrm{eV}$ (Supplementary Fig. 3c, d). The Schottky barrier height is measured as $\sim 501 \pm 26$ $\mathrm{meV}$ in Fig. $2 \mathrm{~d}$ by the variable temperature study (Supplementary Fig. 4), and is the same as the largest Schottky barrier height of $0.5 \mathrm{eV}$ in the reported all 2D Schottky junctions ${ }^{32}$. In the Schottky-Mott rule, the Schottky barrier height in the Schottky junction is defined $\mathrm{as}^{2,3}$ :

$$
\Phi_{\mathrm{SB}}=\Phi_{M}-\chi_{S}
$$

where $\Phi_{M}$ and $\chi_{S}$ are the work function of the metal electrode and the electron affinity of the n-type semiconductors, respectively. The $\chi_{S}$ of monolayer $\mathrm{MoS}_{2}$ previously reported is $\sim 4.3 \mathrm{eV}$ (ref. ${ }^{28,32}$ ), implying the theoretical Schottky barrier height $\Phi_{\mathrm{SB}}$ of the $1 \mathrm{~T}^{\prime}-\mathrm{MoTe}_{2} / \mathrm{MoS}_{2}$ Schottky junction should be $\sim 0.53 \mathrm{eV}$. The experimental and theoretical values are close, suggesting that our constructed $1 \mathrm{~T}^{\prime}-\mathrm{MoTe}_{2} / \mathrm{MoS}_{2}$ Schottky junctions are excellent and largely follow the Schottky-Mott rule.

The work function of Pd is $\sim 5.3 \mathrm{eV}$ (ref. ${ }^{2}$ ), which is larger than that of $\sim 4.83 \mathrm{eV}$ of $1 \mathrm{~T}^{\prime}-\mathrm{MoTe}_{2}$. It should be noted that this work function of $1 \mathrm{~T}^{\prime}-\mathrm{MoTe}_{2}$ was measured under the atmosphere environment and may be slightly different from the true vacuum value. According to the Schottky-Mott rule, the Pd/MoS Schottky junction should possess a larger Schottky barrier height than the $1 \mathrm{~T}^{\prime}-\mathrm{MoTe}_{2} / \mathrm{MoS}_{2}$ Schottky junction. However, the Schottky barrier height of the $1 \mathrm{~T}^{\prime}-\mathrm{MoTe}_{2} / \mathrm{MoS}_{2}$ Schottky junction is significantly higher than the reported values of $\sim 0.12 \mathrm{eV}$ of the $\mathrm{Pd} / \mathrm{MoS}_{2}$ Schottky junction in Fig. 2e (ref. ${ }^{2}$ ). The fundamental reason for causing the opposite result is that unlike $3 \mathrm{D}$ metal $\mathrm{Pd}$, the $2 \mathrm{D}$ metal $1 \mathrm{~T}^{\prime}-\mathrm{MoTe}_{2}$ can suppress the strong Fermi pinning effect at the metal-semiconductor interface. In reality, the Schottky barrier heights between most 3D metals and monolayer $\mathrm{MoS}_{2}$ had seriously deviated from the Schottky-Mott rule ${ }^{22,24}$, resulting in the significantly lower Schottky barrier of $0-0.3 \mathrm{eV}$ than the theoretical values in Fig. 2e. Therefore, followed by the formula (1), the ideal value of the slope $S=\left|d \Phi_{\mathrm{SB}} / d \Phi_{M}\right|$ in Fig. 2e is 1 . However, the $S$ value of the Schottky barrier height between 3D metals and monolayer $\mathrm{MoS}_{2}$ is only 0.09 which is much lower than the ideal value, indicating the Fermi levels of the $\mathrm{MoS}_{2}$ in these metal-semiconductor interfaces are severely pinned. 
The Fermi pinning effect is highly related to the defect-induced gap states of the semiconductors, the additional defects and chemical disorders involved by device fabrications, and the metalinduced gap states 2,24 . The benefits of using $2 \mathrm{D}$ metals in suppressing the Fermi pinning effect are as follows. Firstly, lattice structure damages of the channel materials from the 3D metal evaporation are avoided. Secondly, some chemical disorders in the interface will not be involved by various solvents used in lithography processes. Thirdly, unlike 3D metals, $2 \mathrm{D}$ metals can suppress the metal-induced gap states formed from the decaying metallic wavefunction of the 3D metal itself ${ }^{24}$.

To explore the dependence of the Schottky barrier height on the performance of $2 \mathrm{D}$ semiconductor-based diodes, we compared the rectifying performance of the $\mathrm{Pd} / \mathrm{MoS}_{2}$ and $1 \mathrm{~T}^{\prime}-\mathrm{MoTe}_{2} /$ $\mathrm{MoS}_{2}$ diodes. According to the aforementioned discussions, the band diagrams of the two diodes with different Schottky barrier heights are shown in Fig. 2f. The $1 \mathrm{~T}^{\prime}-\mathrm{MoTe}_{2} / \mathrm{MoS}_{2}$ diode shows good rectifying behavior, and its rectifying ratio reached $\sim 101$ at $V_{\mathrm{DS}}= \pm 1 \mathrm{~V}$ (Fig. $2 \mathrm{~g}$ ). While the rectifying ratio of the $\mathrm{Pd} / \mathrm{MoS}_{2}$ diode is only 11 at $V_{\mathrm{DS}}= \pm 1 \mathrm{~V}$, which is still consistent with the reported values of $4-30$ of the $\mathrm{Pd} / \mathrm{MoS}_{2}$ diodes (Supplementary Table 1). This performance comparison shows that a large Schottky barrier height is essential for building a highperformance Schottky diode.

Widening of Schottky barrier width. To construct an excellent 2D semiconductor-based Schottky diode, in addition to a large Schottky barrier height, a spacious Schottky barrier width is also essential. When the Schottky barrier width is very tiny, the tunneling possibility will significantly increase ${ }^{18}$, resulting in the large reverse tunneling current of diodes. The Schottky barrier width $W$ of the Schottky junction can be obtained by following formula ${ }^{3}$ :

$$
W=\sqrt{\frac{2 \varepsilon_{s} V_{b i}}{q N_{A}}}
$$

where $\varepsilon_{s}$ is the dielectric constant of semiconductors, $V_{b i}=$ $\left(\Phi_{M}-\Phi_{S}\right) / q$ is the built-in potential, $q$ is the elementary charge, and $N_{A}=N_{2 \mathrm{D}} / d_{\mathrm{MoS} 2}$ is the body charge concentration of the channel materials. The smaller Schottky barrier width of the $2 \mathrm{D}$ semiconductor-based Schottky diodes can be attributed to the high carrier concentration of $>10^{19} \mathrm{~cm}^{-3}$ of the ultra-thin physical bodies. Generally, the n-type doping of monolayer $\mathrm{MoS}_{2}$ is considered to mainly originate from the electron doping induced by widespread sulfur vacancies (SVs) ${ }^{33,34}$.

To further improve the quality of the $2 \mathrm{D}$ semiconductor-based Schottky junctions and suppress its reverse leakage current (Fig. 3a), our previous acid-induced sulfur vacancy self-healing (SVSH) effect was employed to heal the SVs of the $\mathrm{MoS}_{2}$ in Fig. 3b (ref. ${ }^{28}$ ). The treatment operation details and healing mechanism are also presented later ('Methods' and Supplementary Fig. 5a). Scanning transmission electron microscopy (STEM) shows the S/Mo ratio of monolayer $\mathrm{MoS}_{2}$ has been increased from $\sim 1.85$ to $\sim 1.92$ in Supplementary Fig. $5 \mathrm{~b}-\mathrm{d}$, which are roughly consistent with the results (from $\sim 1.84$ to $\sim 1.93$ ) measured by X-ray photoelectron spectroscopy (XPS) in Supplementary Fig. 6. The removal of SVs makes the threshold voltage of the $\mathrm{MoS}_{2}$ FET close to zero in Supplementary Fig. 7d, illustrating the intrinsic electron concentration is significantly lowered by 3.9 times from $2.51 \times 10^{12}$ to $6.46 \times 10^{11} \mathrm{~cm}^{-2}$. The lowering of electron concentration is double confirmed by the increase of work function from $\sim 4.3$ to $\sim 4.6 \mathrm{eV}$ measured through the ultraviolet photoelectron spectroscopy (UPS) in Supplementary Fig. 7e.
The decrease of the electron concentration $N_{A}$ has efficiently increased the Schottky barrier width of the $1 \mathrm{~T}^{\prime}-\mathrm{MoTe}_{2} / \mathrm{MoS}_{2}$ Schottky junctions from $\sim 2.9$ to $\sim 4.6 \mathrm{~nm}$ (calculations in 'Methods'). As expected, the SVSH effect significantly shrinks the reverse leakage current from $2 \times 10^{-11}$ to $8 \times 10^{-13} \mathrm{~A}$, while roughly maintaining the forward current (Fig. 3c). The $1 \mathrm{~T}^{\prime}-\mathrm{MoTe}_{2} /$ as-prepared $\mathrm{MoS}_{2}$ Schottky junction shows the obvious rectifying behavior and delivers the maximum rectifying ratio of $\sim 1.4 \times 10^{3}$ at $V_{\mathrm{D}}= \pm 2 \mathrm{~V}$ and $V_{\mathrm{G}}=-40 \mathrm{~V}$ in Fig. 3d. This performance is consistent with the general value $\left(10-10^{4}\right)$ of the $2 \mathrm{D}$ semiconductor-based Schottky junctions reported in the literature (Supplementary Table 1). However, the current rectifying ratio of the $1 \mathrm{~T}^{\prime}-\mathrm{MoTe}_{2} /$ healed $\mathrm{MoS}_{2}$ Schottky junction is enhanced from $\sim 364$ times to $\sim 5.1 \times 10^{5}$ at $V_{\mathrm{D}}= \pm 2 \mathrm{~V}$ and $V_{\mathrm{G}}=-40 \mathrm{~V}$ in Fig. $3 \mathrm{~d}$. This ratio is the best performance among monolayer $2 \mathrm{D}$ semiconductor-based Schottky diodes reported to date (Supplementary Table 1). The gatedependent output curves and transfer curves of the Schottky junction before and after the SVSH are presented in Supplementary Fig. 8. To minimize the performance degradations from transferring monolayer $\mathrm{MoS}_{2}$ in the device fabrication, another device design has been abandoned, in which the $\mathrm{MoS}_{2}$ is top on the metallic 1T '-MoTe 2 . Though the abandoned device design can achieve the SV healing of the $\mathrm{MoS}_{2}$ above the $1 \mathrm{~T}^{\prime}-\mathrm{MoTe}_{2}$, the performance degradations from transferring $\mathrm{MoS}_{2}$ may offset the benefits of defect healing.

To further evaluate the rectifying performance of the $1 \mathrm{~T}$ '- $\mathrm{MoTe}_{2} / \mathrm{MoS}_{2}$ Schottky junction diode, an ideality factor $(n)$ was estimated at a small forward bias (here is $0.02-0.35 \mathrm{~V}$, Fig. $3 \mathrm{c}$ ) by fitting to the Schottky diode equation ${ }^{13,28,35}$ :

$$
I_{\mathrm{D}}=I_{\mathrm{S}}\left[\exp \left(\frac{V_{\mathrm{D}}}{n V_{\mathrm{T}}}\right)-1\right]
$$

where $I_{\mathrm{D}}, I_{\mathrm{S}}, V_{\mathrm{D}}$, and $V_{\mathrm{T}}$ denote drain current, reverse leakage current, drain voltage, and thermal voltage, respectively. As the gate voltage sweep from positive to negative in Fig. 3e, the ideality factor derived from the parameters of the fitting equation gradually approaches the perfect limit value of 1 and gain $\sim 1.6$ at $V_{\mathrm{G}}=-40 \mathrm{~V}$ (the fitted lines of other gate voltages in Supplementary Fig. 8a, b), indicating that the Schottky junction probably reaches a near-ideal diode behavior under the dual effect of the atom-vacancy healing and gate doping ${ }^{2,13,25}$. This similar performance improvement effect can be reproduced in dozens of devices. Besides, with gate voltage sweeping from positive to negative, both the rectifying ratios and ideal factors will gain the best performance at some negative gate voltage and then start to degrade. This performance transition may be related to the tradeoff between the resistances of the bare Schottky junction (consist of region $\mathrm{A}, \mathrm{B} 1$, and $\mathrm{B} 2$ in Fig. 3f) and the series resistances of the $\mathrm{MoS}_{2}$ channel (region B3) 36 . The Schottky junction dominates the total resistance of the diode above the transition voltage. While the interference series resistance of the $\mathrm{MoS}_{2}$ channel will gradually increase its proportion in total resistance and replace the Schottky junction to dominate the diode after crossing the transition voltage.

To remove the interferences from electrode contacts of the $\mathrm{MoS}_{2} / \mathrm{Cr}$ and $1 \mathrm{~T}^{\prime}-\mathrm{MoTe}_{2} / \mathrm{Cr}$ interfaces, both $\mathrm{MoS}_{2}$ and $\mathrm{MoTe}_{2}$ transistors before and after the PEDOT:PSS treatment was also fully characterized. The nearly linear current-voltage relationships in the output curves of the $\mathrm{Cr}-\mathrm{MoS}_{2}-\mathrm{Cr}$ transistors before and after the SVSH are observed in Supplementary Fig. 7b, c, indicating that the Ohmic contacts of the $\mathrm{MoS}_{2} / \mathrm{Cr}$ interfaces have not been adversely affected. It should be noted that the $\mathrm{Cr} /$ $\mathrm{MoS}_{2}$ interfaces are not truly Ohmic contacts ${ }^{20}$, and its Schottky barrier heights before and after the SVSH are largely maintained at $\sim 0.1 \mathrm{eV}$ (Supplementary Fig. 9). The Schottky 

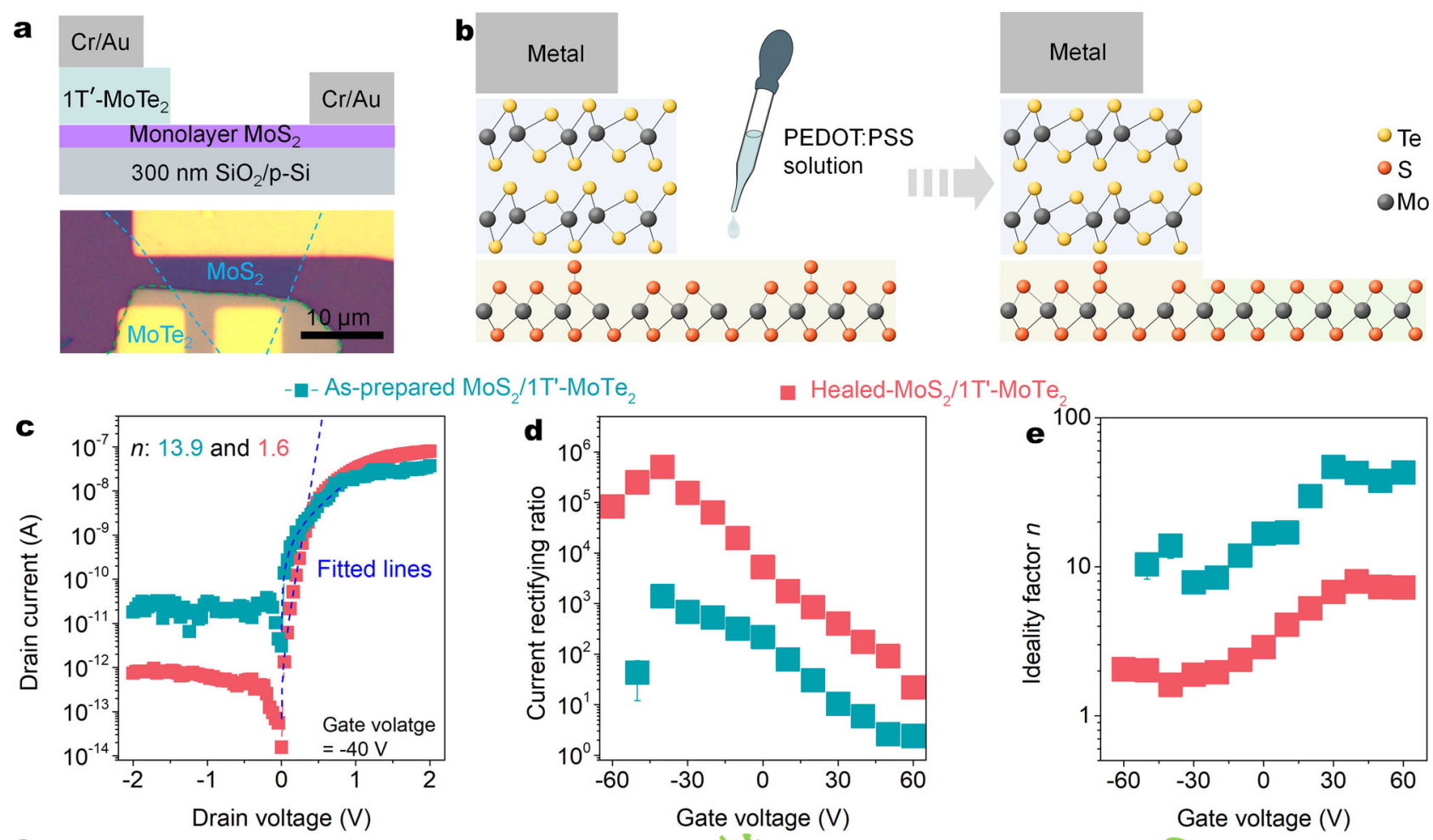

$\mathrm{MoTe}_{2}$

$\mathbf{f}$
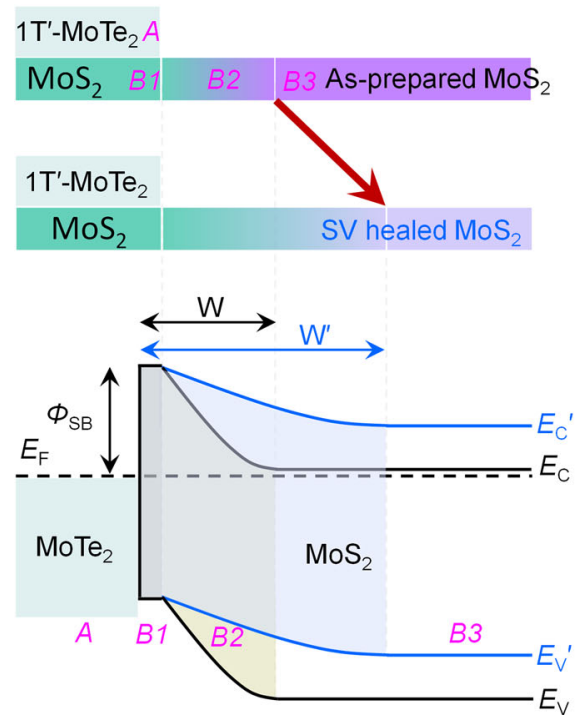
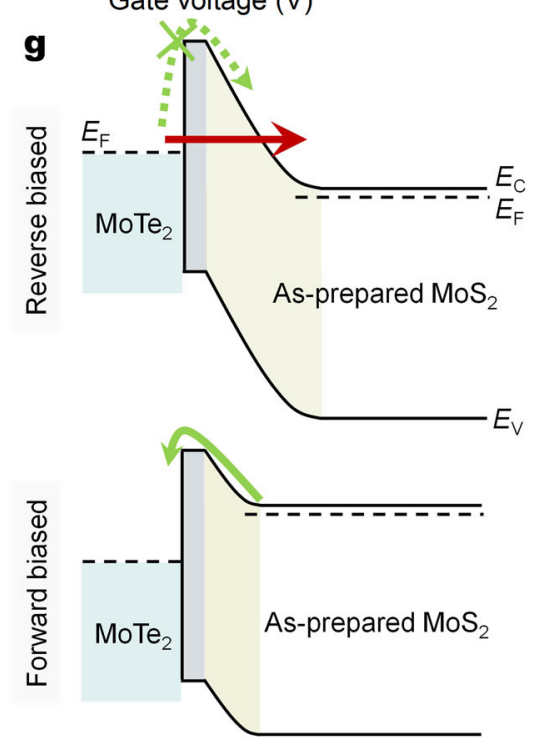
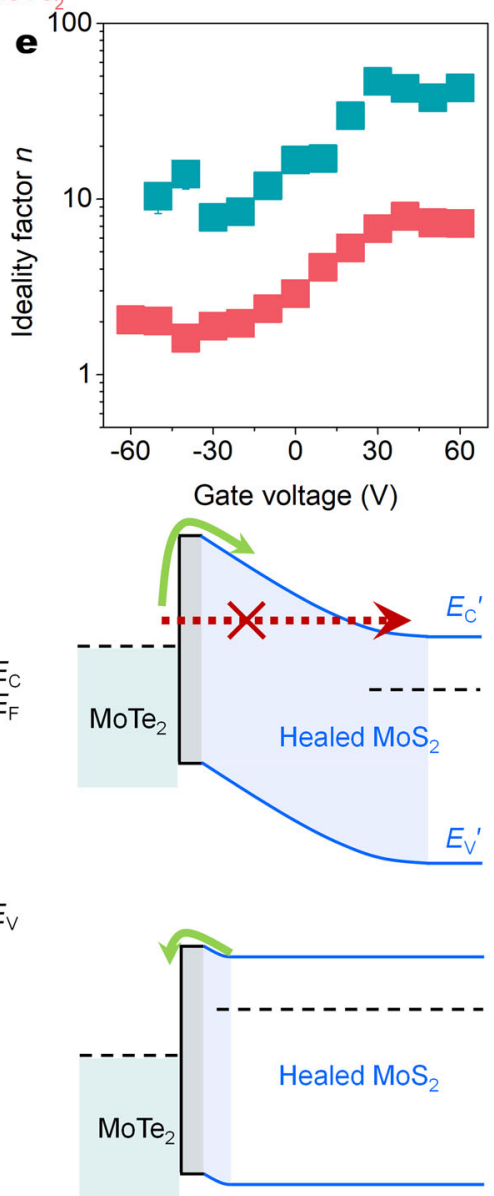

Fig. 3 Giant optimization of the Schottky junction through thickening the Schottky barrier width. a Schematic diagram (top) and optical micrograph (bottom) of $1 \mathrm{~T}^{\prime}-\mathrm{MoTe}_{2} / \mathrm{MoS}_{2}$ Schottky junctions in which the monolayer $\mathrm{MoS}_{2}$ serves as the transport channel, $\mathrm{p}-\mathrm{Si}$ is the control gate, $\mathrm{SiO} \mathrm{O}_{2}$ layer is the gate dielectric, the $1 \mathrm{~T}^{\prime}-\mathrm{MoTe}_{2}$ is the bias drain electrode, and the $\mathrm{Cr} / \mathrm{Au}$ is grounded. $\mathbf{b}$ Schematic diagram of the atomic structure of the $1 \mathrm{~T}^{\prime}-\mathrm{MoTe}_{2} / \mathrm{MoS}_{2}$ Schottky junctions before and after the SVSH. c Output curves in logarithmic scales of the $1 \mathrm{~T}^{\prime}-\mathrm{MoTe}_{2} / \mathrm{MoS}_{2}$ Schottky junctions before and after the SVSH. d, e Gate-dependent current rectifying ratios (d) and ideality factors (e) extracted from Supplementary Fig. 8 before and after the SVSH. $\mathbf{f}$ Device schematic diagrams (top) and band structure diagrams (bottom) before and after the SVSH. The $E_{F}, E_{C_{1}}$ and $E_{V}$ are Fermi level, conduction band, and valence band, respectively. The A, B1, B2, and B3 regions refer to the metal, contacted $\mathrm{MoS}_{2}$, depletion exposed MoS $\mathrm{S}_{2}$ and pure channel MoS 2 . The yellow and blue gradient areas mean the depletion regions of the Schottky junctions. It should be noted that to simplify the band structure, the tunneling barriers originated from the vdWs gap between $1 \mathrm{~T}^{\prime}-\mathrm{MoTe}_{2}$ and $\mathrm{MoS}_{2}$ are intentionally removed in the whole manuscript. $\mathbf{g}$ Band structure diagrams of the twostate devices at forward and reverse biased voltages. The green and red arrows (solid/dashed: main/almost-impossible style) indicate the thermionic emission current and thermally assisted tunneling current, respectively. The movement direction of an electron during reverse (forward) biased voltage is from the $\mathrm{MoTe}_{2}$ to the $\mathrm{MoS}_{2}\left(\mathrm{MoS}_{2}\right.$ to $\left.\mathrm{MoTe}_{2}\right)$.

barriers of the $\mathrm{Cr} / \mathrm{MoS}_{2}$ interfaces are believed to be highly related to the formation of $\mathrm{CrS}_{\mathrm{x}}$ (ref. ${ }^{37}$ ). On the other hand, compared to the untreated one, the treated $1 \mathrm{~T}^{\prime}-\mathrm{MoTe}_{2}$ transistor still maintains a linear current-voltage relationship and constant conductance in Supplementary Fig. 7f-h, indicating that the variations on the $1 \mathrm{~T}^{\prime}-\mathrm{MoTe}_{2}$ side from the acid treatment are almost negligible. Therefore, the main action object of the PEDOT:PSS treatment is the metal-semiconductor interface of the $1 \mathrm{~T}^{\prime}-\mathrm{MoTe}_{2} / \mathrm{MoS}_{2}$ Schottky junction, rather than the other electrode contacts. 
While reducing the electron doping of the SVs of the covered $\mathrm{MoS}_{2}$ (region B2) in Fig. 3f only will efficiently enlarge the depletion width of the Schottky barrier but will not significantly alter the Schottky barrier height $\Phi_{\mathrm{SB}}$. The variable temperature transport studies show the Schottky barrier heights $\Phi_{\mathrm{SB}}$ before and after treatment are calculated as $501 \pm 26$ and $510 \pm 19 \mathrm{meV}$ in Supplementary Fig. 4, suggesting the SVSH effect can not largely alter the Schottky barrier height $\Phi_{\mathrm{SB}}$. On the other hand, if the Schottky barrier height $\Phi_{\mathrm{SB}}$ is considered to be significantly enhanced by the SVSH effect, both the forward and reverse biased currents will be significantly reduced ${ }^{38}$. However, the forwardbiased currents are nearly unchanged or even increased in the partial negative voltage region in Supplementary Fig. $8 \mathrm{c}$ right. Besides, previous studies showed that not doping the covered region $\mathrm{B} 1$ but doping the exposed region $\mathrm{B} 2$ (Fig. $3 \mathrm{f}$ ) could also significantly modulate the Schottky barrier width ${ }^{26}$. In reality, even if the monolayer $\mathrm{MoS}_{2}$ of the covered region B1 (Fig. 3f) was healed, the improvement effect induced by the SVSH on the rectifying behavior of the $1 \mathrm{~T}^{\prime}-\mathrm{MoTe}_{2} / \mathrm{MoS}_{2}$ Schottky diode would not be significantly changed (Supplementary Fig. 10).

Improvement mechanism of the rectifying performance. So how does enlarging the Schottky barrier width improve the rectifying behavior? Based on this band structure transformation of the Schottky junction in Fig. 3f, the depletion region width of the asprepared Schottky junction under reverse bias is too narrow to easily generate large tunneling current Fig. 3g. When the Schottky barrier height remains roughly constant in Fig. $3 g$, enlarging the Schottky barrier width can transform the charge injection style under reverse bias from thermionic emission to thermionic field emission (also call thermally assisted tunneling) ${ }^{17}$. Thus, different from the $1 \mathrm{~T}^{\prime}-\mathrm{MoTe}_{2}$ /as-prepared $\mathrm{MoS}_{2}$ Schottky junction, the reverse leakage currents of the optimized Schottky junctions are significantly suppressed. But in the forward bias, an electron only needs to pass through the Schottky barrier with a small built-in potential to reach the metal electrode $1 \mathrm{~T}^{\prime}-\mathrm{MoTe}_{2}$ in Fig. $3 \mathrm{~g}$ bottom. On the other hand, as forward bias is added, the resistance of the bare Schottky junction decreases, the negative effect of the series resistance of the $\mathrm{MoS}_{2}$ channel (region B3) becomes comparable. To put it simply, the total resistance of the $1 \mathrm{~T}^{\prime}-\mathrm{MoTe}_{2} /$ healed $\mathrm{MoS}_{2}$ Schottky junction in the forward bias can be presumably assumed to be divided into the large $\mathrm{MoS}_{2}$ series resistance (region B3) and small bare Schottky junction resistance (region A, B1, and B2). While the total resistance of the $1 \mathrm{~T}^{\prime}-\mathrm{MoTe}_{2}$ /as-prepared $\mathrm{MoS}_{2}$ Schottky junction is the sum of the small $\mathrm{MoS}_{2}$ series resistance and large bare Schottky junction resistance in Fig. $3 \mathrm{~g}$ bottom-left. In this case, the total resistance of the two Schottky junctions in the forward bias may be close in the most $1 \mathrm{~T}^{\prime}-\mathrm{MoTe}_{2} / \mathrm{MoS}_{2}$ Schottky junctions, which has been confirmed by the two similar forward currents in Fig. 3c and Supplementary Fig. 8d. Based on the performance survey of the dozens of Schottky junctions, the differences between the two forward currents of the as-prepared and healed devices are fluctuated by the $\mathrm{MoS}_{2}$ channel length. In a word, the decline of $E_{F}$ induced by the SVSH can greatly reduce the reverse leakage current, roughly maintain the forward current, and finally improve the rectifying ratio and ideal factor.

Optimization of other Schottky junctions. Except for the $1 \mathrm{~T}^{\prime}$ $\mathrm{MoTe}_{2} / \mathrm{MoS}_{2}$ Schottky diode, the performance optimization strategy induced by the vacancy healing can also be generalized to other $\mathrm{MoS}_{2}$-based diodes. The monolayer $\mathrm{MoS}_{2}$ Schottky diodes with the $\mathrm{Pd}$ and $\mathrm{Cr}$ asymmetric metal electrodes after the solution treatment can only exhibit a maximum rectifying ratio of $\sim 2.9 \times 10^{3}$ in Fig. $4 \mathrm{a}$, which are considerably worse than that of the $1 \mathrm{~T}^{\prime}-\mathrm{MoTe}_{2} /$ healed $\mathrm{MoS}_{2}$ Schottky junctions in Fig. 3d. The poor performance of the $\mathrm{Pd} / \mathrm{MoS}_{2}$ Schottky junction can be partially attributed to its small Schottky barrier height of $\sim 0.12 \mathrm{eV}$ (ref. ${ }^{2}$ ), which is lower than the value of $\sim 0.5 \mathrm{eV}$ of the $1 \mathrm{~T}^{\prime}$ $\mathrm{MoTe}_{2} /$ healed $\mathrm{MoS}_{2}$ Schottky junction in Fig. 2d. Nevertheless, this optimized performance of the $\mathrm{Pd} / \mathrm{MoS}_{2}$ Schottky junction is much higher than that $(1 \sim 100)$ of similar $\mathrm{MoS}_{2}$-based Schottky junctions with evaporated asymmetric metal electrodes (Supplementary Table 1). As mentioned before, the $\mathrm{Pd} / \mathrm{MoS}_{2}$ Schottky junctions may be suffered from the severe Fermi pinning effect in the interface between the evaporated $\mathrm{Pd}$ electrode and the monolayer $\mathrm{MoS}_{2}$. Thus, the origin of the superior rectifying behaviors of the $1 \mathrm{~T}^{\prime}-\mathrm{MoTe}_{2} /$ healed- $\mathrm{MoS}_{2}$ Schottky junctions can be attributed to the dual effect with the large Schottky barrier height and the spacious Schottky barrier width.

Similar to the $1 \mathrm{~T}^{\prime}-\mathrm{MoTe}_{2} / \mathrm{MoS}_{2}$ and $\mathrm{Pd} / \mathrm{MoS}_{2}$ Schottky junction, the rectifying ratios of the $1 \mathrm{~T}-\mathrm{PtSe} \mathrm{S}_{2} / \mathrm{MoS}_{2}$ also were systemically enhanced by the SVSH in Fig. 4b. Due to the similar layered structure between transition metal dichalcogenides (TMDCs) materials, the acid-induced vacancy healing effect has been confirmed to apply to other TMDCs semiconductors ${ }^{39}$. Thus, we also generalize the performance optimization strategy to other TMDCs-based Schottky junctions (Supplementary Fig. 11 and Fig. 4c, d). Similarly, the vacancy healing effect can also increase the rectifying ratios of the $1 \mathrm{~T}^{\prime}-\mathrm{MoTe}_{2} / \mathrm{WS}_{2}$ and $1 \mathrm{~T}$ $\mathrm{PtSe}_{2} / \mathrm{WS}_{2}$ Schottky junctions, which means that their Schottky barrier widths may be greatly enhanced. It should be noted that similar to the $1 \mathrm{~T}^{\prime}-\mathrm{MoTe}_{2}$ film, the $1 \mathrm{~T}-\mathrm{PtSe}_{2}$ transistor still maintains a linear current-voltage relationship and constant metallic conductance in Supplementary Fig. 12.

Photoresponse characterization of the Schottky junctions. Next, we focus on the photoresponse of the $1 \mathrm{~T}^{\prime}-\mathrm{MoTe}_{2} / \mathrm{MoS}_{2}$ Schottky junctions before and after the SVSH and evaluate its performance in photovoltaic detectors in the visible wavelength range. Figure $5 \mathrm{a}$ shows that regardless of the as-prepared device or the healed device, the short-circuit current $I_{\mathrm{SC}}$ and open-circuit voltage $V_{\mathrm{OC}}$ show a monotonic increase with the increase of laser power density. Under the same laser power density, both the $I_{\mathrm{SC}}$ and $V_{\mathrm{OC}}$ are significantly increased by the SVSH effect. As a comparison, the obvious photovoltaic response is not observed in the as-prepared and healed $\mathrm{MoS}_{2}$ transistors (Supplementary Fig. 13), illustrating that the electrode contacts of the $\mathrm{Cr} / \mathrm{MoS}_{2}$ can not donate the photoresponse at zero biased voltage. Besides, the efficient interface charge separation of the $1 \mathrm{~T}^{\prime}-\mathrm{MoTe}_{2} / \mathrm{MoS}_{2}$ Schottky junction has been confirmed by the PL spectra (Supplementary Fig. 14). With the power intensity increasing, the $I_{\mathrm{SC}}$ near linearly increases, and the $V_{\mathrm{OC}}$ will gradually enter the saturated state (Fig. 5b). In the $1 \mathrm{~T}^{\prime}-\mathrm{MoTe}_{2} /$ healed-MoS 2 Schottky junction, a high $I_{\mathrm{SC}}$ of $\sim 22.2 \mathrm{nA}$ and a large $V_{\mathrm{OC}}$ of $\sim 0.19 \mathrm{~V}$ are obtained under the power density of $8.23 \mathrm{~mW} / \mathrm{mm}^{2}$ (junction area of $\sim 671 \mu^{2}$ ). Under the maximum laser power density, the output electrical power density is increased by about 16 times from $\sim 16.7$ to $\sim 270.7 \mu \mathrm{W} / \mathrm{cm}^{2}$ through the SVSH strategy in Fig. $5 c$.

To evaluate the performance improvement of the $\mathrm{MoTe}_{2} / \mathrm{MoS}_{2}$ photodetector, the photoresponsivity and external quantum efficiency (EQE) are systemically characterized. The photoresponsivity is defined as $R=I_{\mathrm{ph}} /\left(P_{\mathrm{in}} \cdot A\right)$, where $I_{\mathrm{ph}}$ is the photocurrent, $P_{\text {in }}$ is the incident laser power density, and $A$ is the junction area of the $1 \mathrm{~T}^{\prime}-\mathrm{MoTe}_{2} / \mathrm{MoS}_{2}$ diode. EQE is the ratio of collected charge carriers to incident photons and can be calculated by EQE $=\left(I_{\mathrm{sc}} \cdot h \cdot \mathrm{c}\right) /\left(e \cdot \lambda \cdot P_{\text {in }} \cdot A\right)$, where $h, \mathrm{c}$, and $\lambda$ are the Planck's constant, the speed of light, and the wavelength of the incident laser, respectively ${ }^{40}$. At laser power density of $\sim 1.9 \mathrm{~mW} / \mathrm{mm}^{2}$ (the laser spot size is $\sim 2.25 \mu \mathrm{m}^{2}$ ), the 

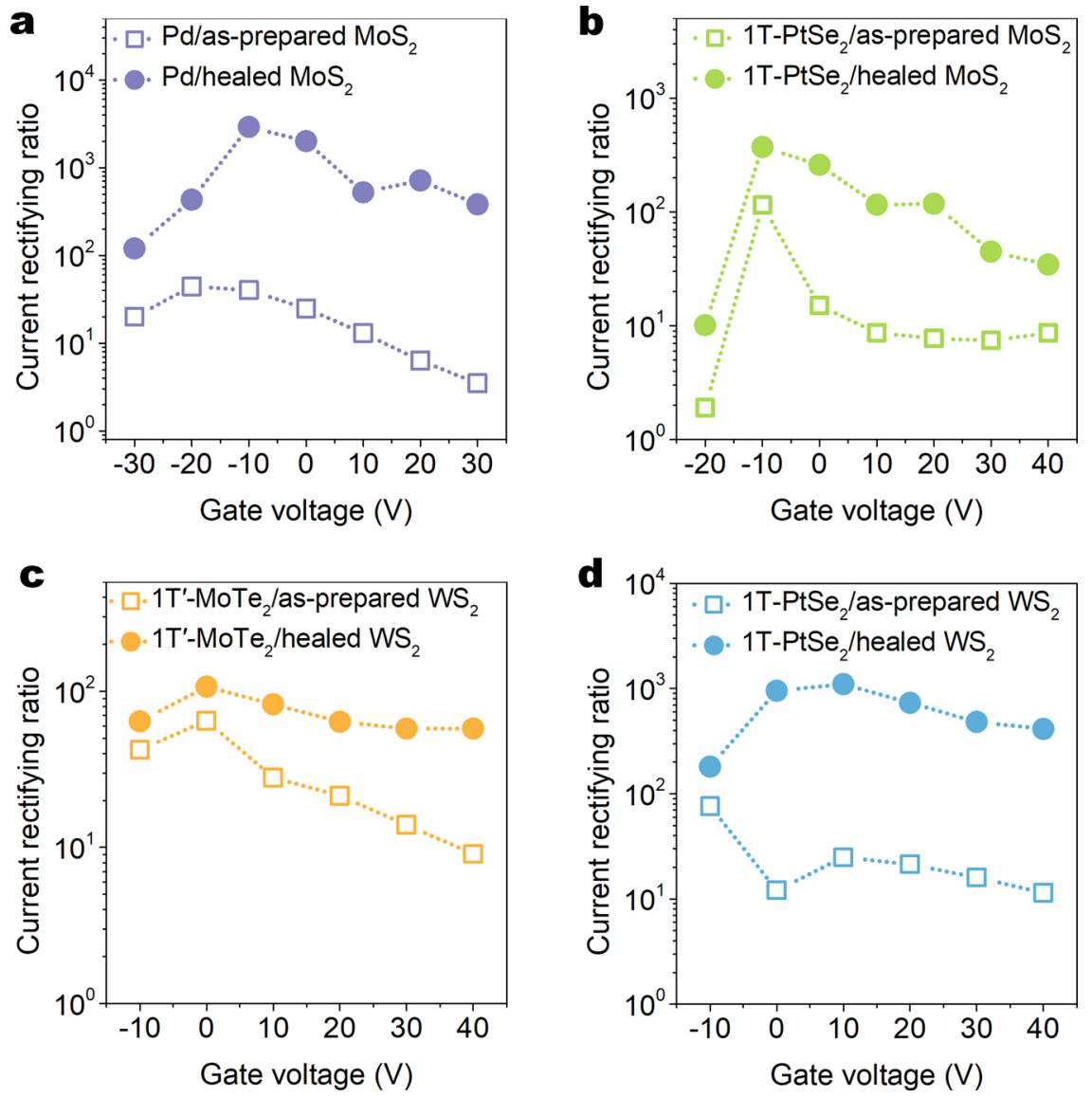

Fig. 4 Generalization of performance optimization strategy of thickening Schottky barrier width. a-d Gate-dependent rectifying ratios of the Pd/MoS 2 (a), $1 \mathrm{~T}-\mathrm{PtSe} \mathrm{S}_{2} / \mathrm{MoS}_{2}(\mathbf{b}), 1 \mathrm{~T}^{\prime}-\mathrm{MoTe}_{2} / \mathrm{WS}_{2}(\mathbf{c})$, and $1 \mathrm{~T}-\mathrm{PtSe}_{2} / \mathrm{WS}_{2}$ (d) metal-semiconductor diodes extracted from Supplementary Fig. 11 before and after the SVSH. It should be noted that both $1 \mathrm{~T}^{\prime}-\mathrm{MoTe}_{2}$ and $1 \mathrm{~T}-\mathrm{PtSe}_{2}$ are multilayer over $10 \mathrm{~nm}$ and behave metallic characteristics to the only function as electrodes.

photoresponsivities of the as-prepared and healed devices are $\sim 59 / 88 \mathrm{~mA} / \mathrm{W}$ and EQE of $\sim 13 / \sim 20 \%$ in Fig. $5 \mathrm{~d}$.

This improvement in photoresponse performance can be attributed to the SVSH effect which greatly enlarges the Schottky barrier width, meaning that the photoresponse area is increased in Fig. 5e, $\mathrm{f}$. The second possible reason is that the sub-bandgap defect states of SVs will trap the photo-generated electrons and promote non-radiative recombination in Fig. 5e, leading to lower $I_{\mathrm{SC}}$ and $V_{\mathrm{OC}}\left(\right.$ ref. ${ }^{41}$ ). Thirdly, a neglected but reliable reason is that the removal of electron doping induced by the SVSH effect greatly improves the light absorption efficiency of monolayer $\mathrm{MoS}_{2}$ to form more photocarriers ${ }^{42,43}$. The electron doping removal induced by gate doping also can enhance the $I_{\mathrm{SC}}$ and $V_{\text {OC }}$ of the $\mathrm{MoS}_{2}$ Schottky junction ${ }^{2}$, while our improvement strategy of defect healing is gate-free and doesn't rely on the external gate voltage.

\section{Discussion}

In conclusion, a near-ideal $1 \mathrm{~T}^{\prime}-\mathrm{MoTe}_{2} / \mathrm{MoS}_{2}$ Schottky diode with a large Schottky barrier height was successfully fabricated by stacking the $2 \mathrm{D}$ metallic $1 \mathrm{~T}^{\prime}-\mathrm{MoTe}_{2}$ and the semiconducting monolayer $\mathrm{MoS}_{2}$. We systemically demonstrate that besides the large Schottky barrier height, thickening the tiny Schottky barrier width can further improve their performance of 2D Schottky junctions by healing the original defects in the $2 \mathrm{D}$ semiconductor counterparts. We further confirm that this Schottky barrier optimization strategy is generally applicable to other Schottky diodes, including $1 \mathrm{~T}-\mathrm{PtSe}_{2} / \mathrm{MoS}_{2}, 1 \mathrm{~T}^{\prime}-\mathrm{MoTe}_{2} / \mathrm{WS}_{2}, 1 \mathrm{~T}-\mathrm{PtSe}_{2} /$
$\mathrm{WS}_{2}$, and $\mathrm{Pd} / \mathrm{MoS}_{2}$. This work provides a proof-of-concept performance optimization strategy for building high-performance Schottky diodes.

\section{Methods}

Growth of monolayer $\mathbf{M o S}_{\mathbf{2}}$. The monolayer $\mathrm{MoS}_{2}$ films were grown by the atmospheric pressure oxygen-assisted CVD method ${ }^{28,35} . \mathrm{MoO}_{3}$ (Sigma-Aldrich, $\geq$ $99.5 \%$ purity) and sulfur (Sigma-Aldrich, $\geq 99.5 \%$ purity) were applied as the precursor and reactant materials, respectively. $\mathrm{MoO}_{3}$ powder $(10 \mathrm{mg})$ was placed in a quartz boat at the center of a single zone furnace (Tianjin Zhonghuan Electrical Furnace Co., Ltd.). A $2 \times 2 \mathrm{~cm}^{2} \mathrm{Si}$ substrates with $300 \mathrm{~nm} \mathrm{SiO}_{2}$ (from Silicon Valley Microelectronics, Inc.) were put face down at top of the $\mathrm{MoO}_{3}$ powder. $\mathrm{S}$ powder was heated to $170^{\circ} \mathrm{C}$ by the home-made heating belt and carried through a highpurity Ar flow of $500 \mathrm{sccm}$. The experiments were implemented at a reaction temperature of $850^{\circ} \mathrm{C}$ for $30 \mathrm{~min}$. When the temperature reached the set value of $850^{\circ} \mathrm{C}$, the oxygen of $\sim 1.2 \mathrm{sccm}$ was introduced as the auxiliary growth gas and it was kept for $30 \mathrm{~min}$. Finally, the monolayer $\mathrm{MoS}_{2}$ samples were cooled naturally under S vapor to minimize SVs.

Thermal CVD growth of $\mathbf{W S}_{\mathbf{2}}$. The $\mathrm{WS}_{2}$ films were grown by the typical thermal CVD process ${ }^{44}$. To prepare the $\mathrm{WS}_{2}$ flake single crystals, an alumina boat loaded with $\mathrm{WS}_{2}$ (Alfa Aesar, $99.8 \%$ purity) powder $(3-5 g$ ), was placed into the middle heating zone of the single zone furnace (from Therm craft XST-2-0-12-MINI-240). The $\mathrm{Si}$ substrates (with $300 \mathrm{~nm} \mathrm{SiO}{ }_{2}$ ) were placed on the quartz boat around the outlet of the furnace. The deposition was performed at the temperature of $\sim 1180^{\circ} \mathrm{C}$ and $150 \mathrm{sccm}$ high-purity Ar flow for 1-3 min.

Material preparation and device construction. The metal phase $1 \mathrm{~T}^{\prime}-\mathrm{MoTe}_{2}$ and $1 \mathrm{~T}-\mathrm{PtSe}_{2}$ films were obtained by mechanical exfoliation of the $1 \mathrm{~T}^{\prime}-\mathrm{MoTe}_{2}$ (from Hefei Kejing Co.) and $1 \mathrm{~T}-\mathrm{PtSe}_{2}$ (from $2 \mathrm{D}$ Semiconductors) bulks. The devices were constructed by stacking CVD-grown monolayer $\mathrm{MoS}_{2}$ and exfoliated $1 \mathrm{~T}^{\prime}-\mathrm{MoTe}_{2}$ flakes with the accurate transfer platform (Metatest, E1-T). We used a standard electron beam lithography (EBL) to pattern source/drain contacts of $\mathrm{MoS}_{2}$ and $1 \mathrm{~T}^{\prime}$ - 
a

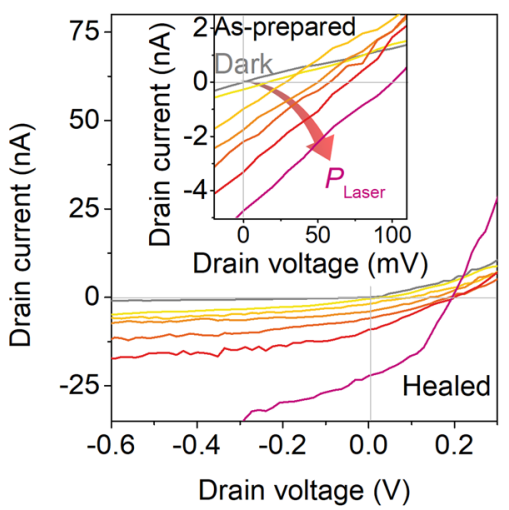

b

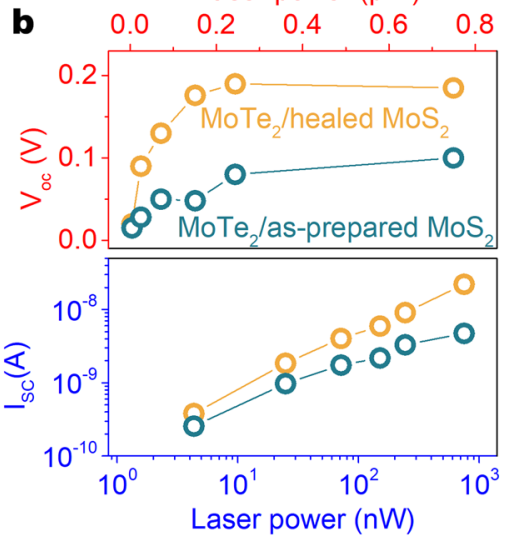

$\mathbf{e}$

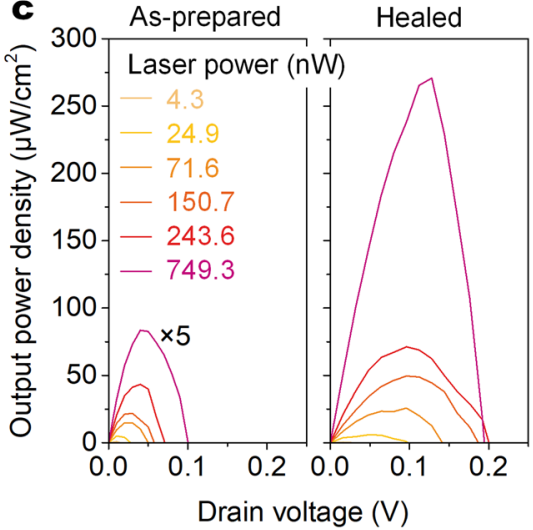

f

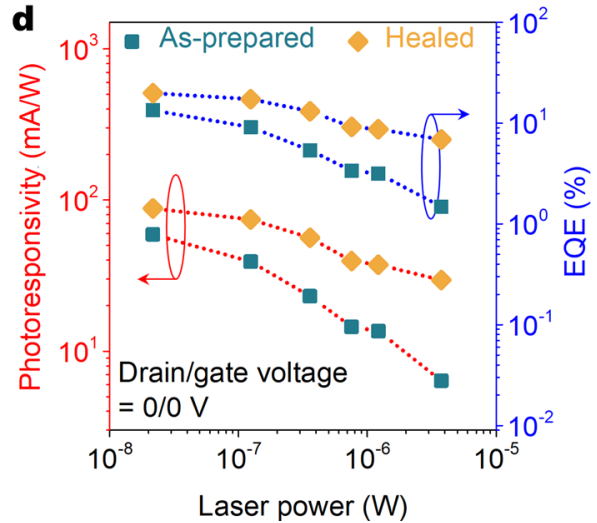

$\mathrm{MoTe}_{2}$ /as-prepared $\mathrm{MoS}_{2}$ (n type)

$\mathrm{MoTe}_{2} /$ healed $\mathrm{MoS}_{2}\left(\mathrm{n}^{-}\right.$type)
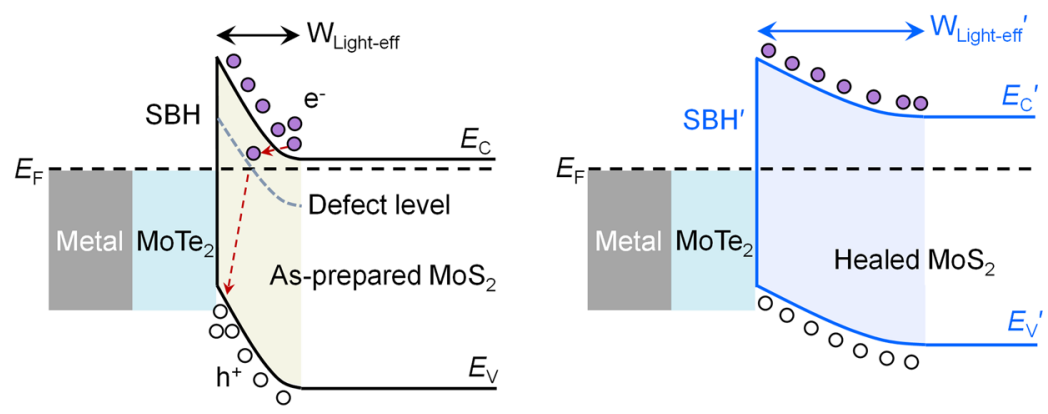

Fig. 5 Photoresponse optimization of the $\mathbf{1 T}^{\prime}-\mathbf{M o T e} \mathbf{2}_{\mathbf{2}} / \mathbf{M o S}_{\mathbf{2}}$ Schottky junction photodiodes. a Output curves of the $1 \mathrm{~T}^{\prime}-\mathrm{MoTe}_{2} / \mathrm{healed}^{\mathrm{MoS}} \mathrm{S}_{2}$ and $1 \mathrm{~T}^{\prime}-\mathrm{MoTe}_{2}$ /as-prepared $\mathrm{MoS}_{2}$ (inset) Schottky junctions in dark and under $532 \mathrm{~nm}$ light irradiation with various laser power densities $\left(P_{\text {Laser }}\right)$. The gate voltages before and after the SVSH are zero. b Short circuit current $\left(I_{\mathrm{SC}}\right)$ and open-circuit voltage $\left(V_{\mathrm{OC}}\right)$ as a function of $P_{\text {Laser }}$ extracted from (a). c Output electrical power of the as-prepared (left) and healed (right) devices as a function of drain-source voltage. $\mathbf{d} P_{\text {Laser }}$-dependent photoresponsivities (red) and EQE (blue) at zero bias. e, $\mathbf{f}$ Band structure diagrams of the $1 \mathrm{~T}^{\prime}-\mathrm{MoTe}_{2} / \mathrm{MoS}_{2}$ Schottky junctions before (e) and after (f) the SVSH under light irradiation. The purple and hollow circles represent electrons and holes, respectively. The red dashed arrow refers to the electron movement direction. The yellow and blue gradient areas refer to the depletion regions of the Schottky junctions. $W_{\text {Light-eff }}$ refers to the efficient light absorption width of the Schottky junction.

$\mathrm{MoTe}_{2}$ FETs, followed by thermal beam evaporation of 10/50-nm-thick $\mathrm{Cr} / \mathrm{Au}$ electrodes and lift-off. The metallization was performed by a thermal evaporation system (KYKY Technology), and the thermal evaporation pressure was $1-5 \times 10^{-5}$ $\mathrm{Pa}$. Especially, our electrode contact optimization for CVD-grown monolayer $\mathrm{MoS}_{2}$ FETs was the mild Argon plasma etching which can remove residual polymethyl methacrylate (PMMA, A8, Mircochem Inc.) and produce a small amount of SVs. The plasma etching parameters (from Tailong Electronics RIE 100) are the radiofrequency $(13.56 \mathrm{MHz})$ power of $5 \mathrm{~W}$, the Ar flow of $10 \mathrm{sccm}$, and the treatment duration of $35-45 \mathrm{~s}$. Both the $\sim 15 / 45 \mathrm{~nm} \mathrm{Pd} / \mathrm{Au}$ electrodes and the $\sim 15 / 45 \mathrm{~nm} \mathrm{Cr} /$ $\mathrm{Au}$ electrodes in the Schottky junction diodes of the asymmetric metal electrodes were deposited by thermal evaporation.

Acid solution treatment for sulfur vacancy self-healing (SVSH). Firstly, the $\mathrm{MoS}_{2}$ sample was immersed in the undiluted poly(3,4-ethylenedioxythiophene):poly(4styrenesulfonate) (PEDOT:PSS) solution (Sigma-Aldrich, $1.0 \mathrm{wt} \%$ ) at room temperature, after standing for $5 \mathrm{~min}$, and then immersed in plenty of deionized (DI) water to wash the water-soluble PEDOT:PSS solution for $30 \mathrm{~min}$. The quality of the DI water, which was used to rinse the samples $\left(\sim 0.8 \times 0.8 \mathrm{~cm}^{2} \mathrm{Si}\right.$ substrates), is $>200+>200 \mathrm{~mL}$ to the twice cleaning. Further, the residual deionized water was dried with nitrogen flow. Both Raman spectra and gas chromatography-mass spectrometry (GC-MS) can prove that there is no residual PEDOT:PSS in the $1 \mathrm{~T}^{\prime}-\mathrm{MoTe}_{2} /$ healed $\mathrm{MoS}_{2}$ Schottky diodes (Supplementary Figs. $7 \mathrm{f}$ and 15 ).

PL and Raman measurements. PL and Raman spectrum measurements were performed with confocal microscopy (JY-HR800 or Renishaw inVia Qontor) under $532 \mathrm{~nm}$ laser at room temperature and in the ambient atmosphere. It should be noted that to reduce the measurement error of the $1 \mathrm{~T}^{\prime}-\mathrm{MoTe}_{2}$ film from strong laser irradiation, both the PMMA package and the weak laser power density $\left(<5 \mathrm{~mW} / \mu \mathrm{m}^{2}\right)$ are simultaneously arranged ${ }^{45}$.
AFM and KPFM measurement. The AFM and KPFM images were obtained via the Bruker Icon AFM system. An AFM tip (SCM-PIT-V2, Bruker Nano Inc., USA) was selected to probe the topography and thickness of the samples under the peakforce working mode, while the AM-KPFM measurements were measured under the tapping mode via the same tip of SCM-PIT-V2 with a resonant frequency of $75 \mathrm{kHz}$, a tip radius of $25 \mathrm{~nm}$, a Pt-Ir coated conductive tip, a tip-sample height of $100 \mathrm{~nm}$, and the ac bias of $0.5 \mathrm{~V}$ on a grounded substrate. These tests were performed in an atmospheric environment.

Calculation of Schottky barrier width $\boldsymbol{W}$. The Schottky barrier width $W$ of the Schottky junction can be obtained by Eq. (2) ${ }^{3}$. We use a relative dielectric constant of 3.3 for $\mathrm{MoS}_{2}\left(\right.$ ref. $\left.{ }^{46}\right)$. The $\Phi_{M}$ of $1 \mathrm{~T}^{\prime}-\mathrm{MoTe}_{2}$ and the $\Phi_{S}$ of $1 \mathrm{H}-\mathrm{MoS}_{2}$ before and after the SVSH effect can be extracted from Supplementary Fig. 3c, d and Supplementary Fig. 6e. The $d_{\mathrm{Mos} 2}$ is the thickness of $0.65 \mathrm{~nm}$ of monolayer $\mathrm{MoS}_{2}$. Thus, the Schottky barrier widths $W$ before and after the SVSH effect are $\sim 2.9$ and $\sim 4.6 \mathrm{~nm}$, respectively.

STEM measurement. STEM samples were prepared using a standard PMMAbased transfer, where the samples were spin coated and soaked in a $\mathrm{KOH}$ solution (5 wt\%). Subsequently, acetone (AR, from RCI Labscan Limited) and isopropyl alcohol (AR, from Aladdin) were used to clean the TEM grid, allowing for the suspension of the films in the solution and the transfer to TEM grids. All STEM samples were baked at $160^{\circ} \mathrm{C}$ for $5 \mathrm{~h}$ under vacuum (the pressure was $<1 \mathrm{~Pa}$ ) before the microscopy experiment and characterized on a JEM-ARM200F TEM operating at $80 \mathrm{kV}$. The STEM images were prepared by partially filtering out the direct spot in the FFT of the image, which helps to remove the contrast generated by the surface contaminations (Supplementary Fig. 5b, c top). After these filtering processes, the STEM images are converted into the plain text signal, in which the signal of the Mo atoms was accurately eliminated by a home-made program. Finally, the program-processed data was produced into the filtered STEM images (Supplementary Fig. 5b, c bottom) by using an Origin Pro 9.0. It should be noted 
that to reduce the interference of ex-situ measurement, the CVD-grown monolayer $\mathrm{MoS}_{2}$ samples in the STEM measurement were originated from the same $\mathrm{SiO}_{2} / \mathrm{Si}$ substrate. For example, a $2 \times 2 \mathrm{~cm}^{2} \mathrm{Si}$ substrates with CVD-grown monolayer $\mathrm{MoS}_{2}$ were evenly cut into two groups. Generally, the defect concentrations of the two sets of samples can be roughly considered the same.

XPS measurement. XPS was carried out using an ESCALab250 electron spectrometer from Thermo Scientific Corporation with monochromatic $150 \mathrm{~W} \mathrm{Al} \mathrm{Ka}$ radiation $(\mathrm{h} v=1486.7 \mathrm{eV})$. The take-off angle for XPS was $90^{\circ}$, and the pass energy for the narrow scan was $30 \mathrm{eV}$. The base pressure was about $6.5 \times 10^{-10} \mathrm{mbar}$. The analyzer of the spectrometer was calibrated using sputter cleaned $\mathrm{Au}, \mathrm{Cu}$, and $\mathrm{Ag}$ foils, as is outlined in the GB/T 19500-2004 standard ${ }^{47}$. In this calibration method, the standard calibrated binding energies of the $\mathrm{Au}, \mathrm{Cu}$, and $\mathrm{Ag}$ standard samples are $\mathrm{Au} 4 f_{7 / 2}$ of $84.0 \pm 0.1 \mathrm{eV}, \mathrm{Cu} 2 p_{3 / 2}$ of $932.7 \pm 0.1 \mathrm{eV}$, and $\mathrm{Ag} 3 d_{5 / 2}$ of $368.3 \pm$ $0.1 \mathrm{eV}$, respectively. To compensate for sample charging, the XPS spectra were calibrated using the Si $2 p^{3 / 2}$ peak of the $\mathrm{SiO}_{2}$ substrate at $103.6 \mathrm{eV}$ as a reference. For XPS peak analysis and deconvolution, the software Avantage 4.15 was employed, where a mixed Gaussian-Lorentzian function and an active Shirley background were used for peak fitting. The S/Mo ratios were determined from the integrated areas of the $\mathrm{S} 2 p$ and Mo $3 d$ peaks factored by their corresponding relative sensitivity factors of 0.668 and 3.321. The error in the S/Mo ratios was obtained from the peak fitting residuals.

UPS measurement. UPS curves were obtained in an ultrahigh vacuum chamber using a helium lamp source emitting (AXIS ULTRA DLD). The ultraviolet light source used was He I $(21.22 \mathrm{eV})$ that has not been monochromated. The basic vacuum of the analysis chamber during the UPS analysis and testing of this equipment was $3.0 \times 10^{-8}$ Torr. The biased voltage during the test was $-9 \mathrm{~V}$.

GC-MS measurement. GC-MS was conducted on an Agilent 7890A/5975C installed with an HP-5MS column $(30 \mathrm{~m} \times 0.25 \mathrm{~mm} \times 0.25 \mu \mathrm{m})$. The temperatures of both inlet and detection were set at $300^{\circ} \mathrm{C}$. The initial temperature of the oven was set at $30^{\circ} \mathrm{C}$ and kept for $1 \mathrm{~min}$, and then was programmed from 30 to $250^{\circ} \mathrm{C}$ with a heating rate of $10^{\circ} \mathrm{C} / \mathrm{min}$, and finally was retained at $250^{\circ} \mathrm{C}$ for additional $5 \mathrm{~min}$. This last increase was to clean the column from any residues. The MS was set as the positive ion model with the mass-to-charge searching region from 20 to 1024. The preparation process of the sample as follows: Firstly, after the 1T '- $\mathrm{MoTe}_{2} / \mathrm{MoS}_{2}$ Schottky diodes were constructed on the $\mathrm{SiO}_{2} / \mathrm{Si}$ substrate, the $\mathrm{Si}$ substrate was immersed in the absolute ethanol (AR, from Honeywell International Inc.) solution of $3 \mathrm{~mL}$ for $2 \mathrm{~h}$. Secondly, remove and blow-dry with nitrogen. After the sample was soaked in the PEDOT:PSS solution for $5 \mathrm{~min}$, was rinsed it twice with plenty of deionized water $(>200+>200 \mathrm{~mL})$ and was dried with nitrogen flow. Thirdly, repeat step \#1. The two different absolute ethanol solutions before and after the PEDOT:PSS treatment were extracted $1 \mu \mathrm{L}$ for the GC-MS test.

Electrical measurement. The electrical characteristics were implemented by a semiconductor analysis system (Keithley 4200 and B2902 A). Electrical measurements were performed in a vacuum probe station (Lakeshore TTP4) with liquid nitrogen and base pressure $\sim 10^{-5}$ Torr.

\section{Data availability}

All data supporting this study and its findings are available within the article and its Supplementary Information or from the corresponding author upon reasonable request.

\section{Code availability}

The codes that support the findings of this study are available from the corresponding author upon reasonable request.

Received: 10 November 2020; Accepted: 17 February 2021;

Published online: 09 March 2021

\section{References}

1. Kappera, R. et al. Phase-engineered low-resistance contacts for ultrathin $\mathrm{MoS}_{2}$ transistors. Nat. Mater. 13, 1128-1134 (2014).

2. Liu, Y. et al. Approaching the Schottky-Mott limit in van der Waals metalsemiconductor junctions. Nature 557, 696-700 (2018).

3. Neamen, D. A. Semiconductor Physics and Devices: Basic Principles (McGrawHill, 2012).

4. $\mathrm{Xu}, \mathrm{Y}$. et al. Contacts between two- and three-dimensional materials: ohmic, Schottky, and p-n heterojunctions. ACS Nano 10, 4895-4919 (2016).

5. Di Bartolomeo, A. Graphene Schottky diodes: An experimental review of the rectifying graphene/semiconductor heterojunction. Phys. Rep. 606, 1-58 (2016).
6. Liu, Y. et al. Van der Waals heterostructures and devices. Nat. Rev. Mater. 1, 16042 (2016).

7. Liu, Y., Huang, Y. \& Duan, X. Van der Waals integration before and beyond two-dimensional materials. Nature 567, 323-333 (2019).

8. Li, J. et al. General synthesis of two-dimensional van der Waals heterostructure arrays. Nature 579, 368-374 (2020).

9. Ubrig, N. et al. Design of van der Waals interfaces for broad-spectrum optoelectronics. Nat. Mater. 19, 299-304 (2020).

10. Zhang, X. et al. Two-dimensional $\mathrm{MoS}_{2}$-enabled flexible rectenna for Wi-Fiband wireless energy harvesting. Nature 566, 368-372 (2019).

11. $\mathrm{Hu}, \mathrm{S}$. et al. Au-InSe van der Waals Schottky junctions with ultralow reverse current and high photosensitivity. Nanoscale 12, 4094-4100 (2020).

12. Cheng, R. et al. High-performance, multifunctional devices based on asymmetric van der Waals heterostructures. Nat. Electron. 1, 356-361 (2018).

13. Jin, Y. et al. Van der Waals homojunction: ideal p-n diode behavior in $\mathrm{MoSe}_{2}$. Adv. Mater. 27, 5534-5540 (2015)

14. Li, Z., Chen, J., Dhall, R. \& Cronin, S. B. Highly efficient, high speed vertical photodiodes based on few-layer $\mathrm{MoS}_{2}$. 2D Mater. 4, 015004 (2016).

15. Miao, J. et al. Gate-tunable semiconductor heterojunctions from $2 \mathrm{D} / 3 \mathrm{D}$ van der Waals interfaces. Nano Lett. 20, 2907-2915 (2020).

16. Chhowalla, M., Jena, D. \& Zhang, H. Two-dimensional semiconductors for transistors. Nat. Rev. Mater. 1, 16052 (2016).

17. Allain, A., Kang, J., Banerjee, K. \& Kis, A. Electrical contacts to twodimensional semiconductors. Nat. Mater. 14, 1195-1205 (2015).

18. Schulman, D. S., Arnold, A. J. \& Das, S. Contact engineering for 2D materials and devices. Chem. Soc. Rev. 47, 3037-3058 (2018).

19. Kim, C. et al. Fermi level pinning at electrical metal contacts of monolayer molybdenum dichalcogenides. ACS Nano 11, 1588-1596 (2017).

20. Das, S., Chen, H. Y., Penumatcha, A. V. \& Appenzeller, J. High performance multilayer $\mathrm{MoS}_{2}$ transistors with scandium contacts. Nano Lett. 13, 100-105 (2013).

21. Heine, V. Theory of surface states. Phys. Rev. 138, A1689-A1696 (1965).

22. Song, S. et al. Wafer-scale production of patterned transition metal ditelluride layers for two-dimensional metal-semiconductor contacts at the SchottkyMott limit. Nat. Electron. 3, 207-215 (2020).

23. Leong, W. S. et al. Synthetic lateral metal-semiconductor heterostructures of transition metal disulfides. J. Am. Chem. Soc. 140, 12354-12358 (2018).

24. Liu, Y., Stradins, P. \& Wei, S. H. Van der Waals metal-semiconductor junction: weak Fermi level pinning enables effective tuning of Schottky barrier. Sci. Adv. 2, e1600069 (2016).

25. LaGasse, S. W., Dhakras, P., Watanabe, K., Taniguchi, T. \& Lee, J. U. Gatetunable graphene-WSe $\mathrm{W}_{2}$ heterojunctions at the Schottky-Mott limit. Adv. Mater. 31, e1901392 (2019).

26. Kiriya, D., Tosun, M., Zhao, P., Kang, J. S. \& Javey, A. Air-stable surface charge transfer doping of $\mathrm{MoS}_{2}$ by benzyl viologen. J. Am. Chem. Soc. 136, 7853-7856 (2014).

27. Yang, L. et al. Chloride molecular doping technique on $2 \mathrm{D}$ materials: $\mathrm{WS}_{2}$ and $\mathrm{MoS}_{2}$. Nano Lett. 14, 6275-6280 (2014).

28. Zhang, X. et al. Poly(4-styrenesulfonate)-induced sulfur vacancy self-healing strategy for monolayer $\mathrm{MoS}_{2}$ homojunction photodiode. Nat. Commun. 8, 15881 (2017).

29. Amani, M. et al. Near-unity photoluminescence quantum yield in $\mathrm{MoS}_{2}$. Science 350, 1065-1068 (2015).

30. Beams, R. et al. Characterization of few-layer $1 \mathrm{~T}^{\prime}-\mathrm{MoTe}_{2}$ by polarizationresolved second harmonic generation and Raman Scattering. ACS Nano 10, 9626-9636 (2016).

31. Keum, D. H. et al. Bandgap opening in few-layered monoclinic $\mathrm{MoTe}_{2}$. Nat. Phys. 11, 482-486 (2015).

32. Shin, H. G. et al. Vertical and in-plane current devices using $\mathrm{NbS}_{2} / \mathrm{n}-\mathrm{MoS}_{2}$ van der Waals Schottky junction and graphene contact. Nano Lett. 18, 1937-1945 (2018).

33. McDonnell, S., Addou, R., Buie, C., Wallace, R. M. \& Hinkle, C. L. Defectdominated doping and contact resistance in $\mathrm{MoS}_{2}$. ACS Nano 8, 2880-2888 (2014).

34. Zhang, X. et al. Hidden vacancy benefit in monolayer 2D semiconductors. Adv. Mater. 33, e2007051 (2021).

35. Gao, L. et al. Defect-engineered atomically thin $\mathrm{MoS}_{2}$ homogeneous electronics for logic inverters. Adv. Mater. 32, e1906646 (2020).

36. Aubry, V. \& Meyer, F. Schottky diodes with high series resistance: Limitations of forward I-V methods. J. Appl. Phys. 76, 7973-7984 (1994).

37. Smyth, C. M., Addou, R., McDonnell, S., Hinkle, C. L. \& Wallace, R. M Contact metal- $\mathrm{MoS}_{2}$ interfacial reactions and potential implications on $\mathrm{MoS}_{2}-$ based device performance. J. Phys. Chem. C 120, 14719-14729 (2016).

38. Yang, H. et al. Graphene barristor, a triode device with a gate-controlled Schottky barrier. Science 336, 1140-1143 (2012).

39. Amani, M. et al. Recombination kinetics and effects of superacid treatment in sulfur- and selenium-based transition metal dichalcogenides. Nano Lett. 16, 2786-2791 (2016) 
40. Wu, F. et al. High efficiency and fast van der Waals hetero-photodiodes with a unilateral depletion region. Nat. Commun. 10, 4664 (2019).

41. Yang, X. et al. Hydroiodic acid additive enhanced the performance and stability of PbS-QDs solar cells via suppressing hydroxyl ligand. Nanomicro Lett. 12, 37 (2020).

42. Mak, K. F. et al. Tightly bound trions in monolayer $\mathrm{MoS}_{2}$. Nat. Mater. 12, 207-211 (2013).

43. Zheng, X. et al. Defect passivation in hybrid perovskite solar cells using quaternary ammonium halide anions and cations. Nat. Energy 2, 17102 (2017).

44. Duan, X. et al. Ultrafast growth of large single crystals of monolayer $\mathrm{WS}_{2}$ and $\mathrm{WSe}_{2}$. Natl Sci. Rev. 7, 737-744 (2020).

45. Cho, S. et al. Phase patterning for ohmic homojunction contact in $\mathrm{MoTe}_{2}$. Science 349, 625-628 (2015).

46. Yoon, Y., Ganapathi, K. \& Salahuddin, S. How good can monolayer $\mathrm{MoS}_{2}$ transistors be? Nano Lett. 11, 3768-3773 (2011).

47. General Rules for X-ray Photoelectron Spectroscopic Analysis Method (accessed Fabruary 2021); https://www.chinesestandard.net/PDF/English.aspx/ GBT19500-2004.

\section{Acknowledgements}

This work was supported by the National Natural Science Foundation of China (Grant Nos. 51991340, 51991342, 51972022, 51722203, 51672026), the Overseas Expertise Introduction Projects for Discipline Innovation (Grant No. B14003), the National Key Research and Development Program of China (Grant Nos. 2018YFA0703503, 2016YFA0202701), the Natural Science Foundation of Beijing Municipality (Grant No. Z180011), and the Fundamental Research Funds for the Central Universities (Grant No. FRF-TP-19-025A3)

\section{Author contributions}

X.Z., Z.Z., and Y.Z. initiated and supervised the project. L.G., Y.L., and B.L. deposited $\mathrm{MoS}_{2}$ monolayers by CVD. X.Z. and B.L. performed device fabrication, data collection, and analysis. J.D., H.Y., M.H., and L.G. assisted in carrying out the film fabrication and characterizations. J.X., Y.L., and L.G. assisted in the device performance measurements. Q.L., Z.K., and B.L. performed part of the Raman, PL, UPS, and XPS characterization.
X.Z. and H.Y. finished the GC-MS, AFM, and KPFM test. L.G. and X.L. carried out STEM experiments. X.Z., Z.Z., and Y.Z. co-wrote the manuscript. All authors discussed the results and commented on the manuscript.

\section{Competing interests}

The authors declare no competing interests.

\section{Additional information}

Supplementary information The online version contains supplementary material available at https://doi.org/10.1038/s41467-021-21861-6.

Correspondence and requests for materials should be addressed to Z.Z. or Y.Z.

Peer review information Nature Communications thanks the anonymous reviewer(s) for their contribution to the peer review of this work.

Reprints and permission information is available at http://www.nature.com/reprints

Publisher's note Springer Nature remains neutral with regard to jurisdictional claims in published maps and institutional affiliations.

(c) (i) Open Access This article is licensed under a Creative Commons Attribution 4.0 International License, which permits use, sharing, adaptation, distribution and reproduction in any medium or format, as long as you give appropriate credit to the original author(s) and the source, provide a link to the Creative Commons license, and indicate if changes were made. The images or other third party material in this article are included in the article's Creative Commons license, unless indicated otherwise in a credit line to the material. If material is not included in the article's Creative Commons license and your intended use is not permitted by statutory regulation or exceeds the permitted use, you will need to obtain permission directly from the copyright holder. To view a copy of this license, visit http://creativecommons.org/ licenses/by/4.0/.

(C) The Author(s) 2021 